\title{
Collisions of antiprotons with hydrogen molecular ions
}

\author{
Armin Lühr and Alejandro Saenz \\ Institut für Physik, AG Moderne Optik, Humboldt-Universität zu Berlin, Hausvogteiplatz, 5-7, D-10117 Berlin, Germany
}

(Received 30 June 2009; published 13 August 2009)

\begin{abstract}
Time-dependent close-coupling calculations of the ionization and excitation cross section for antiproton collisions with molecular hydrogen ions are performed in an impact energy range from $0.5 \mathrm{keV}$ to $10 \mathrm{MeV}$. The Born-Oppenheimer and Franck-Condon approximations as well as the impact parameter method are applied in order to describe the target molecule and the collision process. It is shown that three perpendicular orientations of the molecular axis with respect to the trajectory are sufficient to accurately reproduce the ionization cross section calculated by Sakimoto [Phys. Rev. A 71, 062704 (2005)] reducing the numerical effort drastically. The independent-event model is employed to approximate the cross section for double ionization and $\mathrm{H}^{+}$ production in antiproton collisions with $\mathrm{H}_{2}$.
\end{abstract}

DOI: 10.1103/PhysRevA.80.022705

PACS number(s): 34.50.Gb, 34.50.Bw, 25.43. $+\mathrm{t}$

\section{INTRODUCTION}

A remarkable progress in the understanding of interactions between antiprotons $(\bar{p})$ and atoms has been achieved over the last decades. Although the number of antiproton collision experiments [1-7] is limited due to the required effort for the production of low-energy $\bar{p}$, a large amount of theoretical studies employing a variety of different methods have been performed focusing on hydrogen [8-19] and helium [22-30] targets but also other targets such as alkalimetal [31] or argon [32] atoms have been considered. Among these are full quantum-mechanical treatments for $\mathrm{H}$ as [19] and fully-correlated two-electron calculations for $\mathrm{He}$, e.g. $[20,21]$. They provide single-ionization cross sections in agreement with experiment for $E>40 \mathrm{keV}$. For lower energies, however, some open issues still remain in the comparison among the theoretical results and with the experiments. In the case of double ionization of He differences have been observed between proton $(p)$ and antiproton experiments. The measured data for $\bar{p}$ are larger by a factor 2 even for energies around $1 \mathrm{MeV}$, where the single-ionization cross sections for $\bar{p}$ and $p$ are virtually the same, and were reproduced by theoretical calculations $[21,22]$.

For $\bar{p}$ collisions with molecules experimental $\bar{p}+\mathrm{H}_{2}$ data for ionization cross sections $[1,33]$ and for stopping power $[34,35]$ were measured. As for the double ionization of helium targets, a considerable difference in the production of $\mathrm{H}^{+}$ions was observed between $\bar{p}$ and $p[36,37]$ impacts. Theoretically, however, only little has been investigated for $\bar{p}$ impacts on molecular targets. Very recently, the singleionization and single-excitation cross sections [38] as well as the stopping power [39] for $\bar{p}+\mathrm{H}_{2}$ were calculated using a one-electron one-center description of the $\mathrm{H}_{2}$ target [40]. The experimental $\bar{p}$ results could only be reproduced for impact energies $E \geq 90 \mathrm{keV}$. The findings suggest that for lower energies molecular as well as electron-correlation effects become important and have to be considered.

An elaborate calculation of the ionization process for molecular targets was performed recently by Sakimoto [41] being a pioneering work on $\bar{p}+\mathrm{H}_{2}{ }^{+}$collisions. The calculations were performed using a discrete variable representation (DVR) method in which the wave function is directly calcu- lated on grid points constructed from the zeros of orthogonal polynomials. The author examined closely the dependence of the ionization cross section on the internuclear distance of both nuclei and the relative orientation of the molecular axis with respect to the trajectory of the antiproton. Thereby, it was possible to present cross sections for ionization independent of a fixed orientation of the molecular axis and internuclear separation. However, the author considered the effort for these calculations due to the many degrees of freedom as being extremely time-consuming especially in the case that also excitation cross sections are considered. The calculation of the latter was postponed although the employed method is in principle capable of describing excitation.

It is therefore one aim of the present work to reduce the amount of time needed for the computation of $\bar{p}$ collisions with molecules. This is not only important for the determination of $\mathrm{H}_{2}^{+}$excitation cross sections but even more a prerequisite for calculations of more complex systems such as $\bar{p}+\mathrm{H}_{2}$ which are in the focus of the ongoing research. In order to decrease the computational effort a number of different actions are taken into account in the present work. These are an appropriate basis representation using eigenstates of the unperturbed $\mathrm{H}_{2}{ }^{+}$ion, a reduction of the number of different orientations of the molecular axis which are calculated, and the use of the symmetry of the collision system to reduce the number of coupled equations.

The information on the $\mathrm{H}^{+}$production in $\bar{p}+\mathrm{H}_{2}{ }^{+}$collisions can be used for the interpretation of the $\mathrm{H}^{+}$production in $\bar{p}+\mathrm{H}_{2}$ collisions which was measured one and a half decades ago [1] but is still not understood theoretically. In [41] the idea was discussed to use the concept of a two-step sequential ionization process to explain the measured $\bar{p}+\mathrm{H}_{2}$ data in analogy to what was done by Janev et al. [42] and Wehrman et al. [22] in order to describe double ionization in $\bar{p}+\mathrm{He}$ collisions using an effective single-electron description. The underlying idea is that in a first step the target is ionized and one electron is ejected. In a second step the projectile interacts with an ionic rather than a neutral target reducing the probability for double ionization. It turned out that Wehrman et al. were fairly successful with this independent-event (IEV) model in reproducing the measured antiproton double-ionization cross section for He targets by 
using the product of the single-electron probabilities $p_{\text {ion }}^{\mathrm{He}}$ and $p_{\text {ion }}^{\mathrm{He}^{+}}$for ionization of $\mathrm{He}$ and $\mathrm{He}^{+}$, respectively.

In Sec. II the present method and the used approximations as well as relevant symmetries are described. In Sec. III the convergence behavior of the results is studied. Afterward the calculated ionization and excitation cross sections for $\bar{p}$ $+\mathrm{H}_{2}{ }^{+}$are presented and discussed. The obtained data are used to estimate double ionization and $\mathrm{H}^{+}$production in $\bar{p}$ collisions with $\mathrm{H}_{2}$ molecules. Finally, Sec. IV closes with a summary and conclusions. Atomic units are used unless stated otherwise.

\section{METHOD}

\section{A. Target description}

In the Born-Oppenheimer approximation the total wave function for the $\mathrm{H}_{2}{ }^{+}$molecule separates into the product

$$
\widetilde{\psi}\left(\mathbf{r}, \mathbf{R}_{\text {nuc }}\right)=\frac{\chi_{\nu j}\left(R_{\text {nuc }}\right)}{R_{\text {nuc }}} Y_{j}^{m}(\Theta, \Phi) \psi\left(\mathbf{r} ; R_{\text {nuc }}\right),
$$

where $\chi_{\nu j}$ are the eigenfunctions of the molecular vibration, $Y_{j}^{m}$ the spherical harmonics, and $(\nu, j, m)$ the vibrational and rotational quantum numbers. $\mathbf{R}_{\text {nuc }}=\left(R_{\text {nuc }}, \Theta, \Phi\right)$ and $\mathbf{r}$ are the position vectors of the nuclei and the electron, respectively. The wave function $\psi\left(\mathbf{r} ; R_{\text {nuc }}\right)$ satisfies the electronic part of the time-independent Schrödinger equation

$$
\hat{H}_{e} \psi_{N \Pi}^{M}\left(\mathbf{r} ; R_{\text {nuc }}\right)=\epsilon_{N \Pi}^{M}\left(R_{\text {nuc }}\right) \psi_{N \Pi}^{M}\left(\mathbf{r} ; R_{\text {nuc }}\right)
$$

for an unperturbed molecule at a fixed internuclear distance $R_{\text {nuc }}$, where $\hat{H}_{e}$ is the sum of the potential and the electronic part of the kinetic operator. In contrast to atomic targets which are spherical symmetric the two-center monoatomic molecule ion $\mathrm{H}_{2}{ }^{+}$obeys different molecular symmetries. Instead of the atomic quantum numbers the electronic part of the $\mathrm{H}_{2}{ }^{+}$eigenstates $\psi$ can be characterized by $N, M$, and $\Pi$, where $\Pi$ is the permutation symmetry with the values $g$ for gerade and $u$ for ungerade symmetry, $M$ is the projection of the angular momentum on the internuclear axis, and $N$ is the principal quantum number.

For the description of the electronic wave functions

$$
\psi_{N \Pi}^{M}\left(\mathbf{r} ; R_{\mathrm{nuc}}\right)=\sum_{l=l_{\min }(M, \Pi)}^{l_{\max }(\Pi)} \rho_{N l}^{M}\left(r ; R_{\mathrm{nuc}}\right) Y_{l}^{M}(\omega),
$$

a one-center expansion around the midpoint of the internuclear axis is chosen, where $r$ and $\omega$ are the radial and angular variables of the electron, respectively. In the expansion in Eq. (3) only even or odd values of $l$ contribute depending on whether $\Pi$ is gerade or ungerade, respectively, and $l_{\min } \geq M$. Note, $l_{\min }$ and $l_{\max }$ are merely basis-set parameters. The angular part is described with spherical harmonics $Y_{l}^{M}(\omega)$. The $z^{\prime}$ axis of the molecule-fixed space is chosen along the internuclear axis. The radial part $\rho_{N l}^{M}(r)$ is expanded in a $B$-spline basis of the order 8 . The radial equation is solved in a finite box with a radius of 100 a.u. using fixed boundary conditions by what bound as well as discretized continuum states are obtained. The appropriate representation of the continuum is an advantage of the used basis expansion which was already successfully employed before for atomic targets [31].

The electronic structure code which solves Eq. (2) uses a one-center approximation of the molecular potential

$$
V\left(\mathbf{r}, R_{\mathrm{nuc}}\right)=-\frac{1}{\left|\mathbf{r}+\frac{\mathbf{R}_{\mathrm{nuc}}}{2}\right|}-\frac{1}{\left|\mathbf{r}-\frac{\mathbf{R}_{\mathrm{nuc}}}{2}\right|},
$$

which is expanded using the relation found by Legendre

$$
\frac{1}{\left|\mathbf{r}_{1}-\mathbf{r}_{2}\right|}=\sum_{s=0}^{\infty} \tilde{V}_{s}\left(r_{1}, r_{2}\right) P_{s}(\cos \gamma),
$$

where $\gamma$ is the angle between $\mathbf{r}_{1}$ and $\mathbf{r}_{2}$, the $P_{s}$ are the Legendre polynomials and $\tilde{V}_{s}\left(r_{1}, r_{2}\right)$ is given by

$$
\tilde{V}_{s}\left(r_{1}, r_{2}\right)=\left\{\begin{array}{ll}
\frac{r_{1}^{s}}{r_{2}^{(s+1)}} & \text { for } r_{1} \leq r_{2} \\
\frac{r_{2}^{s}}{r_{1}^{(s+1)}} & \text { for } r_{1}>r_{2}
\end{array} .\right.
$$

The expansion in Eq. (5) becomes accurate only in the limit $s \rightarrow \infty$. However, it is known to be applicable using a small $s_{\max }$ as an upper limit of the sum being therefore an expansion parameter. Actually, if the ansatz of Eq. (3) is used for $\psi$ then $s_{\max }=2 l_{\max }$ holds by what $l_{\max }$ becomes the decisive expansion parameter. More details of the code, which was used in order to calculate photon-induced processes [43-45], were discussed in [43]. It is based on an atomic code [46,47], which was frequently applied before, e.g., in calculations of antiproton collisions with atomic targets [31,38,39].

\section{B. Impact parameter approximation}

The collision process is considered in a semiclassical way using the impact parameter method (cf., e.g., Ref. [48]) which is believed to be highly accurate for impact energies $E \gtrsim 1 \mathrm{keV}$. The quantum-mechanically treated electron is exposed to the Coulomb potential of the molecular nuclei as well as the heavy projectile. The latter is assumed to move on a straight classical trajectory $\mathbf{R}(t)=\mathbf{b}+\mathbf{v} t$ given by the impact parameter $\mathbf{b}$ and its velocity $\mathbf{v}$ while $t$ is the time. The space-fixed coordinate system is defined with the $x$ and $z$ axis being parallel to $\mathbf{b}$ and $\mathbf{v}$, respectively.

For a fixed $\mathbf{R}_{\text {nuc }}$ the collision process can be described by the time-dependent Schrödinger equation

$$
i \frac{\partial}{\partial t} \Psi(\mathbf{r}, \mathbf{R}(t))=\left[\hat{H}_{e}+\hat{V}_{\mathrm{int}}(\mathbf{r}, \mathbf{R}(t))\right] \Psi(\mathbf{r}, \mathbf{R}(t)),
$$

where the interaction between the projectile with charge $Z_{p}$ and the target electron is expressed by the time-dependent interaction potential

$$
\hat{V}_{\text {int }}(\mathbf{r}, \mathbf{R}(t))=-\frac{Z_{p}}{|\mathbf{r}-\mathbf{R}(t)|} .
$$

The interaction of the projectile with the nucleus leads only to an overall phase which does not change the total cross 
sections. It is therefore not considered in this study.

The time-dependent scattering wave function

$$
\Psi(r, \mathbf{R}(t))=\sum_{k} c_{k}(\mathbf{R}(t)) \psi_{k}(\mathbf{r}) e^{-i \epsilon_{k} t}
$$

is expanded in the normalized time-independent eigenstates $\psi$ as given in Eq. (3) and $k \equiv N M \Pi$ stands for the quantum numbers needed to label these states. Substitution of Eq. (3) into Eq. (7) and projection with $\psi_{k}$ leads to the usual coupled equations

$$
i \frac{\mathrm{d} c_{k}}{\mathrm{~d} t}=e^{i \epsilon_{k} t} \sum_{j}\left\langle\psi_{k}\left|\hat{V}_{\mathrm{int}}\right| \psi_{j}\right\rangle e^{-i \epsilon_{j} t},
$$

for every trajectory $\mathbf{R}(t)$, i.e., for every impact parameter $b$ and every impact energy $E=(1 / 2) M_{p} v^{2}$, where $M_{p}$ is the projectile mass. The $c_{k}(\mathbf{R}(t))$ depends of course also on (the fixed) $\mathbf{R}_{\text {nuc }}$. The coupled differential equations [Eq. (10)] are integrated in a finite $z$ range -50 a.u. $\leq z=v t \leq 50$ a.u. with the initial conditions $c_{k}\left[\mathbf{R}\left(t_{i}=-50 / v\right)\right]=\delta_{k i}$, i.e., the target is initially in the electronic state $\psi_{i}$.

The probability for a transition into the electronic final state $\psi_{k}$ at $t_{f}=50 / v$ for a fixed $\mathbf{R}_{\text {nuc }}=\left(R_{\text {nuc }}, \Theta, \Phi\right)$ is given by

$$
p_{k}\left(b, E ; R_{\text {nuc }}, \Theta, \Phi\right)=\left|c_{k}\left(b, v, t_{f} ; R_{\text {nuc }}, \Theta, \Phi\right)\right|^{2} .
$$

In accordance with [41], the transition probability

$$
\begin{aligned}
p_{k}(b, E)= & \int\left|\chi_{\nu j}\left(R_{\text {nuc }}\right) Y_{j}^{m}(\Theta, \Phi)\right|^{2} \\
& \times p_{k}\left(b, E ; R_{\text {nuc }}, \Theta, \Phi\right) \sin \Theta \mathrm{d} R_{\text {nuc }} \mathrm{d} \Theta \mathrm{d} \Phi .
\end{aligned}
$$

becomes orientation-independent by integration over $\mathbf{R}_{\text {nuc }}$. The corresponding cross section

$$
\sigma_{k}(E)=2 \pi \int p_{k}(b, E) b \mathrm{~d} b,
$$

can then be obtained by integration over $b$ as it is done for atomic targets which are spherical symmetric. The total cross sections for ionization,

$$
\sigma_{\text {ion }}(E)=\sum_{\epsilon_{k}>0} \sigma_{k}(E),
$$

and for excitation of the target,

$$
\sigma_{\mathrm{exc}}(E)=\sum_{\epsilon_{0}<\epsilon_{k}<0} \sigma_{k}(E),
$$

can be obtained by summing up all partial cross sections into states $k$ [as given in Eq. (13)] with positive energy and all $\sigma_{k}$ for states with negative energy being larger than that of the ground state $\epsilon_{0}$, respectively.

\section{Franck-Condon approximation}

The dependence of the ionization cross section on the internuclear distance $R_{\text {nuc }}$ for $\bar{p}+\mathrm{H}_{2}{ }^{+}$was examined in [41] for the range 1.5 a.u. $\leq R_{\text {nuc }} \leq 3$ a.u. in which the radial distribution $\left|\chi_{\nu j}\right|^{2}$ of the vibrational ground state $\chi_{00}$ is nonnegligible. It has been shown that the dependence of the

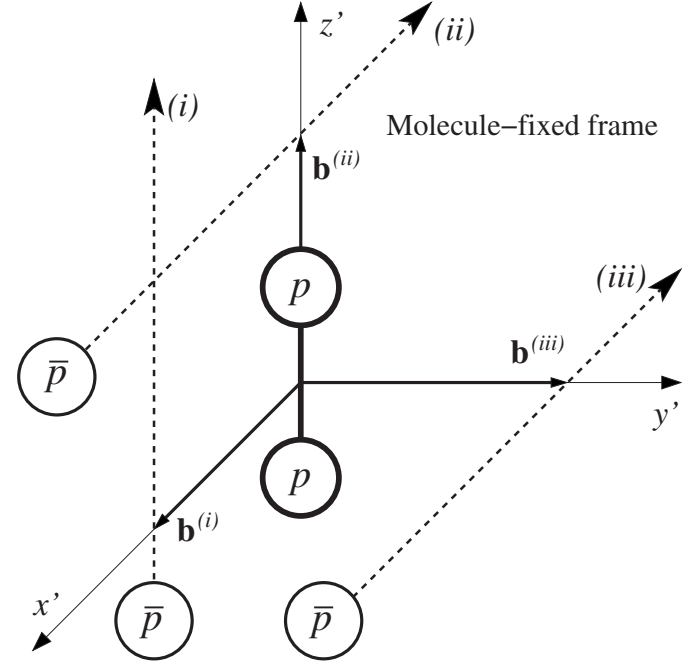

FIG. 1. Sketch of the molecule-fixed frame. The $z^{\prime}$ axis is parallel to the internuclear axis of the $\mathrm{H}_{2}{ }^{+}$. Shown are three trajectories of the $\bar{p}$ and the corresponding impact parameters $\mathbf{b}$ for the orientations (i), (ii), and (iii), which are further explained in the text.

cross sections on $R_{\text {nuc }}$ is approximately linear. A similar dependence of the cross sections on $R_{\text {nuc }}$ was also obtained in calculations for $\bar{p}+\mathrm{H}_{2}$ in [38]. Under the assumption that $\left|\chi_{\nu j}\right|^{2}$ is an even function of $R_{\text {nuc }}-\bar{R}_{\text {nuc }}$ and $\sigma\left(R_{\text {nuc }}\right)$ is linear in $R_{\text {nuc }}$ around $\bar{R}_{\text {nuc }}$ the Franck-Condon (FC) approximation becomes accurate as discussed, e.g., in [49], where $\bar{R}_{\text {nuc }}$ $\equiv\left\langle R_{\text {nuc }}\right\rangle$ is the expectation value of $R_{\text {nuc }}$. Consequently, in [41] the FC results were found to be very close to the exact cross sections obtained by an integration over $R_{\text {nuc }}$ like in Eq. (12).

In what follows the FC approximation is used, i.e., the calculations of the ionization and excitation cross sections are performed for $R_{\text {nuc }}=2.05$ a.u. which is the expectation value for the ground state.

\section{Molecular orientation}

In contrast to atomic targets one set of trajectories in which only $b$ is varied is not sufficient for molecular targets. In the case of a molecule also different angular orientations between the trajectory of the projectile and the molecular axis have to be considered in order to perform the integration in Eq. (12). This leads to a three-dimensional set of trajectories which can be characterized by $\{b, \Theta, \Phi\}$. In the spacefixed frame, defined by $\mathbf{b}$ and $\mathbf{v}$, the position vector of the projectile is $\mathbf{R}(t)=\left(R_{x}, R_{y}, R_{z}\right)=(b, 0, v t)$ and the spherical coordinates of one molecular nucleus are given by $\left(R_{\text {nuc }} / 2, \Theta, \Phi\right)$.

The electronic wave functions $\psi$ in Eq. (3), however, are defined in the molecule-fixed frame in which the $z^{\prime}$ axis is chose to be parallel to the internuclear axis (cf. Fig. 1). Therefore, also the time-dependent calculations of the collision process are performed in the latter frame. Therein, the position vector of the projectile $\mathbf{R}=\left(R_{x^{\prime}}, R_{y^{\prime}}, R_{z^{\prime}}\right)$ can be written as

$$
R_{x^{\prime}}=b \cos \Theta \cos \Phi-v t \sin \Theta,
$$




$$
\begin{gathered}
R_{y^{\prime}}=-b \sin \Phi, \\
R_{z^{\prime}}=b \sin \Theta \cos \Phi+v t \cos \Theta,
\end{gathered}
$$

or be expressed in spherical molecule-fixed coordinates

$$
\begin{gathered}
R=\sqrt{R_{x^{\prime}}^{2}+R_{y^{\prime}}^{2}+R_{z^{\prime}}^{2}}=\sqrt{b^{2}+v^{2} t^{2}}, \\
\theta=\arccos \left(\frac{R_{z^{\prime}}}{R}\right), \\
\phi=\arctan \left(\frac{R_{y^{\prime}}}{R_{x^{\prime}}}\right),
\end{gathered}
$$

where $\phi$ can take all values within the interval $[0,2 \pi]$. Note, in Eqs. (19)-(21) all three spherical coordinates are explicitly dependent on the time as well as on the impact parameter $b$. The angular coordinates $\theta$ and $\phi$ depend also on the relative orientation between the trajectory and the internuclear axis given by $\Theta$ and $\Phi . R=|\mathbf{R}|$ is obviously the same in both frames.

\section{E. Interaction matrix elements}

The matrix elements of the time-dependent interaction potential induced by the moving projectile are similar to those for atomic targets in [31]. However, due to the loss of the spherical symmetry of the target two major differences exist. First, different orientations of the molecular axis lead to different interaction matrix elements. Second, the reduction of symmetries results in other (good) quantum numbers $(M, \Pi)$ and therefore to a different scheme of calculating the matrix elements.

The matrix elements of the interaction potential between the two states $\psi_{N^{\prime} \Pi^{\prime}}^{M^{\prime}}$ and $\psi_{N \Pi}^{M}$ which are required in Eq. (10) are given by

$$
\begin{aligned}
& \left\langle\psi_{N^{\prime} \Pi^{\prime}}^{M^{\prime}}\left|\hat{V}_{\mathrm{int}}\right| \psi_{N \Pi \Pi}^{M}\right\rangle \\
& =\left\langle\psi_{N^{\prime} \Pi}^{M^{\prime}}\left|\sum_{s=0}^{\infty} \sum_{q=-s}^{+s} \frac{4 \pi}{(2 s+1)} \tilde{V}^{s} \mathrm{Y}_{s}^{q^{*}}(\theta, \phi) \mathrm{Y}_{s}^{q}(\omega)\right| \psi_{N \Pi}^{M}\right\rangle \\
& =V_{M^{\prime}, M}^{(\phi)} \sum_{l^{\prime}=l_{\min }^{\prime}}^{l_{\max }^{\prime}} \sum_{l=l_{\min }}^{l_{\max }} \sum_{s=s_{\text {low }}}^{s_{\text {up }}} V_{M^{\prime} N^{\prime} l^{\prime}, M N l, s}^{(R)} V_{M^{\prime} l^{\prime}, M l, s}^{(\theta)} .
\end{aligned}
$$

The three terms $V_{M^{\prime}, M}^{(\phi)}, V_{M^{\prime} N^{\prime} l^{\prime}, M N l, s}^{(R)}$, and $V_{M^{\prime} l^{\prime}, M l, s}^{(\theta)}$ which depend on $\phi, R$, and $\theta$, respectively are defined as

$$
\begin{gathered}
V_{M^{\prime}, M}^{(\phi)}=e^{-i\left(M^{\prime}-M\right) \phi}, \\
V_{M^{\prime} N^{\prime} l^{\prime}, M N l, s}^{(R)}=\left\langle\rho_{N^{\prime} l^{\prime}}^{M^{\prime}}\left|\tilde{V}_{s}(r, R)\right| \rho_{N l}^{M}\right\rangle,
\end{gathered}
$$

$$
\begin{aligned}
V_{M^{\prime} l^{\prime}, M l, s}^{(\theta)}= & (-1)^{M^{\prime} \sqrt{\left(2 l^{\prime}+1\right)(2 l+1)}} \\
& \times \sqrt{\frac{\left[s-\left(M^{\prime}-M\right)\right] !}{\left[s+\left(M^{\prime}-M\right)\right] !} \mathrm{P}_{s}^{\left(M^{\prime}-M\right)}(\cos \theta)} \\
& \times\left(\begin{array}{lll}
l^{\prime} & s & l \\
0 & 0 & 0
\end{array}\right)\left(\begin{array}{ccc}
l^{\prime} & s & l \\
-M^{\prime} & {\left[M^{\prime}-M\right]} & M
\end{array}\right),
\end{aligned}
$$

where the $P_{l}^{m}$ are the associated Legendre polynomials. Due to the Wigner-3j symbols in Eq. (26), $s$ only takes even or odd values depending on whether $s_{\text {up }}=l_{\max }^{\prime}+l_{\max }$ is even or odd. The lower limit of $s$ is determined by $s_{\text {low }}=\max \left(\mid M^{\prime}\right.$ $\left.-M|,| l_{\max }^{\prime}-l_{\max } \mid\right)$. Note, that expression (23) factorizes into two parts which can be determined independently, i.e., $V_{M^{\prime}, M}^{(\phi)}$ and a second term depending on $R$ and $\theta$ in which different $V^{(R)}$ and $V^{(\theta)}$ are mixed by the summations over $l^{\prime}, l$ and $s$. Furthermore, the behavior of the expressions in Eqs. (24)-(26) under exchange of the initial and the final state as well as under a simultaneous change of the signs of $M^{\prime}$ and M

$$
\begin{gathered}
V_{M^{\prime}, M}^{(\phi)}=V_{M, M^{\prime}}^{(\phi) *}=V_{-M^{\prime},-M}^{(\phi) *}=V_{-M,-M^{\prime}}^{(\phi)}, \\
V_{M^{\prime} N^{\prime} l^{\prime}, M N l, s}^{(R)}=V_{-M^{\prime} N^{\prime} l^{\prime},-M N l, s}^{(R)} \\
=V_{M N l, M^{\prime} N^{\prime} l^{\prime}, s}^{(R)}=V_{-M N l,-M^{\prime} N^{\prime} l^{\prime}, s}^{(R)}, \\
V_{M^{\prime} l^{\prime}, M l, s}^{(\theta)}=(-1)^{\left(M^{\prime}-M\right)} V_{-M^{\prime} l^{\prime},-M l, s}^{(\theta)} \\
=V_{M l, M^{\prime} l^{\prime}, s}^{(\theta)}=(-1)^{\left(M^{\prime}-M\right)} V_{-M l,-M^{\prime} l^{\prime}, s}^{(\theta)},
\end{gathered}
$$

can be used to reduce the computational effort.

While $V^{(\theta)}$ and $V^{(\phi)}$ can be determined analytically the radial part $V^{(R)}$ is integrated numerically using quadrature. Furthermore, the number of different $V^{(R)}$ is much larger than those of $V^{(\theta)}$ and $V^{(\phi)}$ since the former depends on all parameters. Therefore, $V^{(R)}$ has to be evaluated efficiently.

\section{F. Symmetries and selection rules}

One aim of the present study is to reduce the computational effort for $\bar{p}+\mathrm{H}_{2}{ }^{+}$collisions drastically. This permits the description of excitation cross sections but it is even more a precondition in view of future calculations for the much more demanding system $\bar{p}+\mathrm{H}_{2}$. As can be seen from Eq. (11) a large number of calculations are necessary in order to perform an integration over the angles $\Theta$ and $\Phi$ in Eq. (12). An alternative approach, though approximate, is to use for fixed $R_{\text {nuc }}, b$, and $E$ an orientationally averaged transition probability defined by

$$
p_{k}=\frac{1}{3}\left[p_{k}(0,0)+p_{k}\left(\frac{\pi}{2}, 0\right)+p_{k}\left(\frac{\pi}{2}, \frac{\pi}{2}\right)\right],
$$

in which only the three perpendicular orientations $(\Theta, \Phi)$ $=(\mathrm{i})(0,0)$, (ii) $(\pi / 2,0)$, (iii) $(\pi / 2, \pi / 2)$ are considered instead of performing the integration in Eq. (12).

In this work it was found that the integrated ionization cross sections by Sakimoto [41]_obtained according to Eq. 
(12) — can be reproduced nicely using_according to Eq. (30) - his results only for the three perpendicular orientations (i), (ii), and (iii). The relative difference of the ionization cross section obtained by integration and by averaging of the probabilities for the three orientations is around $1 \%$ for $E$ $=2 \mathrm{keV}$ and $2 \%$ for $E=100 \mathrm{keV}$. Therefore, in what follows only the three perpendicular orientations (i), (ii), and (iii) are considered, although the present method is capable of arbitrary angular orientations. In Fig. 1 the trajectories for (i), (ii), and (iii) are sketched in the molecule-fixed frame. In the following, the properties of these three trajectories and the symmetries of the according interaction matrix elements in Eq. (23) are discussed.

In (i) the molecule- and space-fixed frame coincide resulting in the same kind of problem as for atomic targets (cf., e.g., $[31,50])$, i.e., $\cos \theta=(v t) / R$ and $\phi \equiv 0$. In (ii) the molecule is oriented parallel to the impact parameter which gives $\phi$ equal to 0 or $\pi$ for $R_{x^{\prime}}>0$ or $R_{x^{\prime}}<0$, respectively, and for $\theta$ one gets $\cos \theta=b / R$. Finally, in (iii) the molecular axis is oriented perpendicular to the collision plane resulting in a time-dependent $\phi=\arctan [b /(v t)]$ while $\theta$ is constant with $\cos \theta=0$.

For (i) and (ii) the azimuthal angle $\phi$ can be considered as constant [58]. As a consequence, the system of coupled equations in Eq. (10) can be transformed in such a way that only positive $M$ quantum numbers have to be treated explicitly when solving the coupled differential equations. Such a transformation was demonstrated in, e.g., Ref. [50]. Alternatively, the angular part of basis states can be described with a combination of spherical harmonics $\left[(-1)^{m} Y_{l}^{m}+Y_{l}^{-m}\right]$ which is solely real as it has been done for atomic targets (cf., e.g., Refs. [31,48]).

In (iii) these simplifications are not possible since $\phi$ is time-dependent. Consequently, positive as well as negative $M$ quantum numbers have to be considered. However, the fact that $\cos \theta \equiv 0$ holds can be exploited. As a consequence, in the interaction matrix elements of $V^{(\theta)}$ in Eq. (26) all odd associated Legendre polynomials, i.e., $s+M^{\prime}+M$ being odd, vanish. As a result a selection rule only allows for transitions in which the parities of the initial and final state differ and the difference of the initial and final $M$ is odd or both parities are equal and the difference of the $M$ is even. In the case that the $\mathrm{H}_{2}{ }^{+}$molecular ion is initially in its ground state only transitions among the symmetry subspaces $(M, \Pi)=(0, g)$, $(1, u),(2, g),(3, u),(4, g), \ldots$ are allowed.

Due to the mentioned symmetries for (i) and (ii) as well as (iii) the eigenstates separate into two sets which can be treated independently since they are not coupled by the matrix elements of the Coulomb interaction [Eq. (23)]. By this the numerical effort can be reduced by nearly a factor 4 . Note, although the time propagation in (i) and (ii) has only to be performed for either $M \geq 0$ or $M \leq 0$ the matrix elements in Eq. (23) have to be computed for negative and positive $M$ which, however, differ only in $V^{(\theta)}$ and $V^{(\phi)}$.

\section{RESULTS}

\section{A. Convergence behavior}

In Tables I-III the variations of the quantities $\sigma_{\text {ion }}(\Theta, \Phi)$ and $\sigma_{\text {exc }}(\Theta, \Phi)$ with respect to the basis-set parameters $N, M$,
TABLE I. Variation of $\sigma_{\text {ion }}(\Theta, \Phi)$ and $\sigma_{\text {exc }}(\Theta, \Phi)$, in units of $10^{-16} \mathrm{~cm}^{2}$, for $\bar{p}+\mathrm{H}_{2}{ }^{+}$with respect to $N$, the number of $B$ splines, while $M=3$ and $\Delta l=2$ are kept constant and $R_{\text {nuc }}=2.0$ a.u.

\begin{tabular}{|c|c|c|c|c|c|c|}
\hline \multirow{3}{*}{$\begin{array}{l}E(\mathrm{keV}) \\
N\end{array}$} & \multicolumn{3}{|c|}{$\sigma_{\text {ion }}(\Theta, \Phi)$} & \multicolumn{3}{|c|}{$\sigma_{\mathrm{exc}}(\Theta, \Phi)$} \\
\hline & 2 & 50 & 250 & 2 & 50 & 250 \\
\hline & \multicolumn{6}{|c|}{$(\Theta, \Phi)=(0,0)$} \\
\hline 14 & 0.0737 & 0.4065 & 0.1867 & 0.8755 & 1.4922 & 0.7251 \\
\hline 21 & 0.1194 & 0.4064 & 0.1856 & 0.8443 & 1.4633 & 0.7172 \\
\hline 30 & 0.1383 & 0.4053 & 0.1869 & 0.8247 & 1.4638 & 0.7177 \\
\hline 35 & 0.1396 & 0.4059 & 0.1872 & 0.8226 & 1.4651 & 0.7187 \\
\hline 50 & 0.1396 & 0.4058 & 0.1871 & 0.8228 & 1.4651 & 0.7186 \\
\hline 65 & 0.1396 & 0.4059 & 0.1871 & 0.8228 & 1.4651 & 0.7187 \\
\hline$N$ & \multicolumn{6}{|c|}{$(\Theta, \Phi)=(\pi / 2,0)$} \\
\hline 14 & 0.0340 & 0.4574 & 0.1980 & 0.3165 & 2.1410 & 1.2231 \\
\hline 21 & 0.0447 & 0.4453 & 0.2023 & 0.2994 & 2.1068 & 1.2137 \\
\hline 30 & 0.0476 & 0.4433 & 0.2031 & 0.2973 & 2.1074 & 1.2127 \\
\hline 35 & 0.0480 & 0.4439 & 0.2032 & 0.2980 & 2.1072 & 1.2127 \\
\hline 50 & 0.0480 & 0.4438 & 0.2031 & 0.2979 & 2.1073 & 1.2127 \\
\hline 65 & 0.0480 & 0.4438 & 0.2031 & 0.2979 & 2.1073 & 1.2127 \\
\hline$N$ & \multicolumn{6}{|c|}{$(\Theta, \Phi)=(\pi / 2, \pi / 2)$} \\
\hline 14 & 0.0280 & 0.2982 & 0.1661 & 0.1892 & 0.7161 & 0.5202 \\
\hline 21 & 0.0393 & 0.2787 & 0.1615 & 0.1709 & 0.7013 & 0.5159 \\
\hline 30 & 0.0439 & 0.2786 & 0.1619 & 0.1670 & 0.7034 & 0.5169 \\
\hline 35 & 0.0444 & 0.2793 & 0.1622 & 0.1675 & 0.7061 & 0.5182 \\
\hline 50 & 0.0444 & 0.2792 & 0.1621 & 0.1675 & 0.7059 & 0.5181 \\
\hline 65 & 0.0444 & 0.2792 & 0.1621 & 0.1675 & 0.7061 & 0.5182 \\
\hline
\end{tabular}

and $\Delta l$ are presented considering the three different orientations $(\Theta, \Phi)=(\mathrm{i})(0,0)$, (ii) $(\pi / 2,0)$, and (iii) $(\pi / 2, \pi / 2)$. The $\sigma_{\text {ion }}(\Theta, \Phi)$ and $\sigma_{\text {exc }}(\Theta, \Phi)$ are defined in accordance with Eq. (14) of Ref. [41] by

$$
\begin{aligned}
& \sigma_{\text {ion }}(\Theta, \Phi)=2 \pi \int p_{\text {ion }}(b ; \Theta, \Phi) b \mathrm{~d} b, \\
& \sigma_{\text {exc }}(\Theta, \Phi)=2 \pi \int p_{\text {exc }}(b ; \Theta, \Phi) b \mathrm{~d} b,
\end{aligned}
$$

where $p_{\text {ion }}$ and $p_{\text {exc }}$ are the probabilities for ionization and excitation, respectively. A fixed internuclear distance is used which is chosen to be $R_{\text {nuc }}=2.0$ a.u. for the convergence study being the equilibrium distance of an $\mathrm{H}_{2}^{+}$molecule. Note, that the $\sigma_{\text {ion }}(\Theta, \Phi)$ and $\sigma_{\text {exc }}(\Theta, \Phi)$ as given in Eqs. (31) and (32) are no measurable quantities and are only defined in order to learn more about the orientational dependence.

Table I shows the excellent convergence behavior of the $\sigma(\Theta, \Phi)$ with respect to the number $N$ of states per $M, \Pi$, and $l$, independently of the impact energy, angular orientation or whether ionization or excitation is considered. A rela- 
TABLE II. Variation of $\sigma_{\text {ion }}(\Theta, \Phi)$ and $\sigma_{\text {exc }}(\Theta, \Phi)$, in units of $10^{-16} \mathrm{~cm}^{2}$, for $\bar{p}+\mathrm{H}_{2}{ }^{+}$with respect to $M$ while $N=50$ and $\Delta l=3$ are kept constant and $R_{\text {nuc }}=2.0$ a.u.

\begin{tabular}{|c|c|c|c|c|c|c|}
\hline \multirow{3}{*}{$\begin{array}{l}E(\mathrm{keV}) \\
M\end{array}$} & \multicolumn{3}{|c|}{$\sigma_{\text {ion }}(\Theta, \Phi)$} & \multicolumn{3}{|c|}{$\sigma_{\mathrm{exc}}(\Theta, \Phi)$} \\
\hline & 2 & 50 & 250 & 2 & 50 & 250 \\
\hline & \multicolumn{6}{|c|}{$(\Theta, \Phi)=(0,0)$} \\
\hline 1 & 0.1371 & 0.4118 & 0.1547 & 0.8046 & 1.4858 & 0.7184 \\
\hline 2 & 0.1353 & 0.4045 & 0.1839 & 0.7936 & 1.4404 & 0.7062 \\
\hline 3 & 0.1350 & 0.4004 & 0.1889 & 0.7916 & 1.4367 & 0.6998 \\
\hline 4 & 0.1354 & 0.3999 & 0.1894 & 0.7923 & 1.4366 & 0.6982 \\
\hline$M$ & \multicolumn{6}{|c|}{$(\Theta, \Phi)=(\pi / 2,0)$} \\
\hline 1 & 0.0497 & 0.4447 & 0.2047 & 0.2787 & 2.1237 & 1.2262 \\
\hline 2 & 0.0465 & 0.4435 & 0.2138 & 0.2875 & 2.0899 & 1.2144 \\
\hline 3 & 0.0458 & 0.4413 & 0.2194 & 0.2890 & 2.0856 & 1.2096 \\
\hline 4 & 0.0460 & 0.4404 & 0.2203 & 0.2898 & 2.0851 & 1.2092 \\
\hline$M$ & \multicolumn{6}{|c|}{$(\Theta, \Phi)=(\pi / 2, \pi / 2)$} \\
\hline 1 & 0.0472 & 0.2019 & 0.1149 & 0.1496 & 0.7817 & 0.4905 \\
\hline 2 & 0.0426 & 0.2573 & 0.1501 & 0.1537 & 0.6984 & 0.4991 \\
\hline 3 & 0.0411 & 0.2704 & 0.1617 & 0.1552 & 0.6666 & 0.4964 \\
\hline 4 & 0.0410 & 0.2711 & 0.1659 & 0.1552 & 0.6592 & 0.4949 \\
\hline
\end{tabular}

tively small value of $N=35$ yields already relative errors $|\Delta \sigma(\Theta, \Phi) / \sigma(\Theta, \Phi)|<0.1 \%$ for all considered $\sigma_{\text {ion }}(\Theta, \Phi)$ and $\sigma_{\text {exc }}(\Theta, \Phi)$ of Table I.

The variation of the $\sigma_{\text {ion }}(\Theta, \Phi)$ and $\sigma_{\text {exc }}(\Theta, \Phi)$ with respect to $M$ in Table II yields relative errors for $M=3$ which are smaller than $0.5 \%$ except for (iii) where these are $2.5 \%$ for $\sigma_{\text {ion }}(\pi / 2, \pi / 2)$ at $E=250 \mathrm{keV}$ and $1.1 \%$ for $\sigma_{\text {ex }}(\pi / 2, \pi / 2)$ at $E=250 \mathrm{keV}$.

A somewhat worse convergence behavior can be observed in Table III for the variation of $\Delta l=\left(l_{\max }-l_{\min }\right) / 2$ which is the number of different $l$ per symmetry subspace $(M, \Pi)$. $\Delta l=3$ gives relative errors less than $1 \%, 1.5 \%$, and $2.5 \%$ for the orientations (i), (ii), and (iii), respectively. Increasing $\Delta l$ to a value of 4 decreases the maximal relative error to $0.6 \%$. In general larger $\Delta l$ are required for lower impact energies. This trend is also known from atomic targets [8,31].

In the calculations of the present results the basis set parameters are chosen to be $(N, M, \Delta l)=(35,3,3)$ for the orientations (i) and (ii) and $(N, M, \Delta l)=(35,4,3)$ for (iii). The size of the basis is in general given by $N \times(2 M+1) \times 2 \Delta l$. Exploiting the symmetries discussed in Sec. II F a total number of 840 and 945 coupled differential equations have to be solved for (i), (ii) and (iii), respectively. A further increase of one of the parameters $N, M$, or $\Delta l$ leads for nearly all energies and orientations to decreasing $\sigma_{\text {ion }}(\Theta, \Phi)$ and $\sigma_{\text {exc }}(\Theta, \Phi)$. Therefore, the present results obtained with fixed sets of $(N, M, \Delta l)$ might be considered as upper bounds to the exact values. An energy cutoff of 25 a.u. is used, i.e., only (continuum) states $\psi_{k}$ with $\epsilon_{k}<25$ a.u. are considered in the expansion of $\Psi$ in Eq. (9).
TABLE III. Variation of $\sigma_{\text {ion }}(\Theta, \Phi)$ and $\sigma_{\text {exc }}(\Theta, \Phi)$, in units of $10^{-16} \mathrm{~cm}^{2}$, for $\bar{p}+\mathrm{H}_{2}^{+}$with respect to $\Delta l=\left(l_{\max }-l_{\min }\right) / 2$ while $N$ $=50$ and $M=3$ are kept constant and $R_{\text {nuc }}=2.0$ a.u.

\begin{tabular}{|c|c|c|c|c|c|c|}
\hline \multirow{3}{*}{$\begin{array}{l}E(\mathrm{keV}) \\
\Delta l\end{array}$} & \multicolumn{3}{|c|}{$\sigma_{\text {ion }}(\Theta, \Phi)$} & \multicolumn{3}{|c|}{$\sigma_{\mathrm{exc}}(\Theta, \Phi)$} \\
\hline & 2 & 50 & 250 & 2 & 50 & 250 \\
\hline & \multicolumn{6}{|c|}{$(\Theta, \Phi)=(0,0)$} \\
\hline 1 & 0.2010 & 0.3311 & 0.1716 & 0.7733 & 1.5828 & 0.7971 \\
\hline 2 & 0.1396 & 0.4058 & 0.1871 & 0.8228 & 1.4651 & 0.7186 \\
\hline 3 & 0.1349 & 0.3977 & 0.1901 & 0.7917 & 1.4374 & 0.6986 \\
\hline 4 & 0.1338 & 0.3938 & 0.1900 & 0.7847 & 1.4314 & 0.6929 \\
\hline 5 & 0.1332 & 0.3929 & 0.1900 & 0.7827 & 1.4295 & 0.6912 \\
\hline
\end{tabular}

\begin{tabular}{lllllll}
$\Delta l$ & \multicolumn{7}{c}{$(\Theta, \Phi)=(\pi / 2,0)$} \\
\hline 1 & 0.0672 & 0.3594 & 0.1252 & 0.3312 & 2.0894 & 1.0544 \\
2 & 0.0480 & 0.4438 & 0.2031 & 0.2979 & 2.1073 & 1.2127 \\
3 & 0.0457 & 0.4382 & 0.2213 & 0.2891 & 2.0859 & 1.2070 \\
4 & 0.0450 & 0.4344 & 0.2243 & 0.2859 & 2.0822 & 1.2023 \\
5 & 0.0449 & 0.4334 & 0.2248 & 0.2848 & 2.0809 & 1.2007
\end{tabular}

\begin{tabular}{lllllll}
\hline$\Delta l$ & \multicolumn{7}{c}{$(\Theta, \Phi)=(\pi / 2, \pi / 2)$} \\
1 & 0.0855 & 0.4475 & 0.1944 & 0.2809 & 1.1539 & 0.7686 \\
2 & 0.0444 & 0.2792 & 0.1621 & 0.1675 & 0.7059 & 0.5181 \\
3 & 0.0411 & 0.2701 & 0.1616 & 0.1552 & 0.6666 & 0.4960 \\
4 & 0.0401 & 0.2664 & 0.1601 & 0.1513 & 0.6559 & 0.4898 \\
5 & 0.0399 & 0.2656 & 0.1599 & 0.1503 & 0.6529 & 0.4881 \\
\hline \hline
\end{tabular}

\section{B. Total cross sections}

Calculations for $\bar{p}$ collisions with $\mathrm{H}_{2}{ }^{+}$are performed within a broad energy range of $0.5 \mathrm{keV} \geq E \geq 10 \mathrm{MeV}$. The three orientations of the molecular axis in the space-fixed frame $(\Theta, \Phi)=(0,0),(\pi / 2,0)$, and $(\pi / 2, \pi / 2)$ are considered. Trajectories for these three directions in the moleculefixed frame are sketched in Fig. 1. The FC approximation is employed throughout with $R_{\text {nuc }}=2.05$ a.u. An orientationally averaged transition probability $p_{k}(b, E)$ is gained according to Eq. (30) from the results for the three orientations. Subsequently, the total ionization and excitation cross sections are obtained as given in Eqs. (13)-(15). The present data for the total ionization and excitation cross sections $\sigma_{\text {ion }}$ and $\sigma_{\text {exc }}$, respectively, are listed for a selection of energies in Table IV.

\section{Ionization}

The orientationally averaged FC cross sections for ionization $\sigma_{\text {ion }}$ are shown in Fig. 2(a) and are compared to results calculated by Sakimoto [41]. The present calculations reproduce the latter results nearly perfectly. From Table IV it can be seen that for $E \leq 50 \mathrm{keV}$ the agreement is better than $1 \%$. For $E=100,200$, and $500 \mathrm{keV}$, the difference is of the order of $2.1 \%, 1.7 \%$, and $0.9 \%$, respectively. The increased differences between the two calculations for the latter three energies might be caused by a reduction of the basis size in [41] for $E>50 \mathrm{keV}$ while it is kept the same in the present work. 
TABLE IV. Ionization and excitation cross sections for antiproton collisions with $\mathrm{H}_{2}{ }^{+}$in $10^{-16} \mathrm{~cm}^{2}$ which are shown in Figs. 2(a) and 2(b), respectively. The results are given for the mean value of the internuclear distance $R_{\text {nuc }}=\left\langle R_{\text {nuc }}\right\rangle=2.05$ a.u. in accordance with the Frank-Condon approximation. The literature data for ionization were calculated by Sakimoto [41].

\begin{tabular}{lccc}
\hline \hline $\begin{array}{l}E \\
(\mathrm{keV})\end{array}$ & $\sigma_{\text {ion }}$ & $\sigma_{\text {ion }}^{\text {lit }}$ & $\sigma_{\text {exc }}$ \\
\hline 1 & 0.036 & & 0.235 \\
2 & 0.077 & 0.078 & 0.445 \\
4 & 0.147 & & 0.785 \\
5 & 0.173 & & 0.909 \\
8 & 0.239 & & 1.176 \\
10 & 0.268 & 0.268 & 1.288 \\
20 & 0.348 & 0.349 & 1.504 \\
40 & 0.383 & & 1.516 \\
50 & 0.379 & 0.380 & 1.475 \\
80 & 0.350 & & 1.373 \\
100 & 0.326 & 0.333 & 1.271 \\
200 & 0.228 & 0.232 & 0.940 \\
400 & 0.135 & & 0.631 \\
500 & 0.112 & 0.113 & 0.538 \\
800 & 0.073 & & 0.392 \\
1000 & 0.060 & & 0.392 \\
2000 & 0.031 & & 0.189 \\
4000 & 0.016 & & 0.102 \\
8000 & 0.008 & & 0.053 \\
\hline \hline
\end{tabular}

As intended in [41] this reduction seems to have less influence on the results with increasing $E$ but is still visible for $E \leq 200 \mathrm{keV}$. The author of [41] also mentions that for $E$ $=100 \mathrm{keV}$ and $(\Theta, \Phi)=(0,0)$ the relative convergence error is largest and not below $2 \%$.

Due to the good agreement between both calculations the following conclusions can be drawn. First, the results by Sakimoto [41] are confirmed by the use of a substantially independent approach. Second, the use of only three orthogonal orientations of the molecular axis seems to be sufficient for the description of the total ionization cross section in $\bar{p}+\mathrm{H}_{2}{ }^{+}$collisions. Consequently, the effort is reduced drastically since a two-dimensional integration over the angles $\Theta$ and $\Phi$ is not performed. Such an integration as given in Eq. (12) requires a sufficient number of supporting points, i.e., calculations, in $\Theta$ and $\Phi$ direction which is of course much larger than three as used in the present approach for simple averaging in Eq. (30).

\section{Excitation}

The orientationally averaged FC cross sections for excitation $\sigma_{\mathrm{exc}}$ are shown in Fig. 2(b). To the best of the authors' knowledge there are no literature data for excitation in $\bar{p}$ $+\mathrm{H}_{2}{ }^{+}$collisions available to compare to. Converged excitation cross sections especially for high $E$ require an extended range of the impact parameter $b$ in comparison to ionization
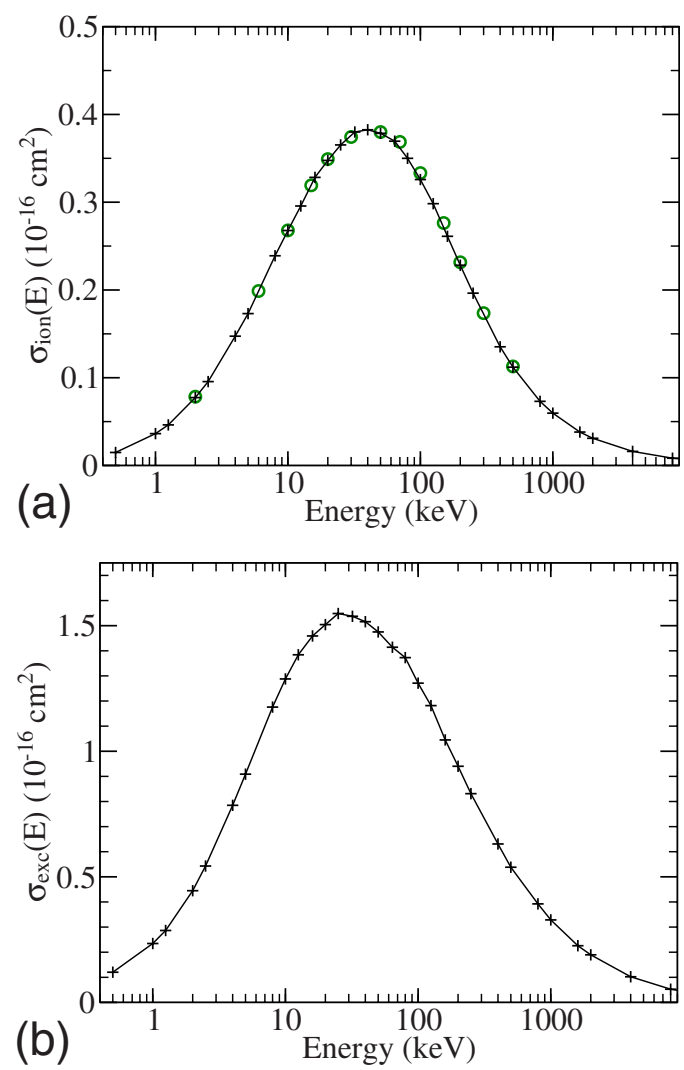

FIG. 2. (Color online) Cross section for $\bar{p}+\mathrm{H}_{2}{ }^{+}$as a function of the antiproton impact energy. Black solid curve with plus, present results (orientationally averaged); green circles, calculation by Sakimoto [41]. (a) Ionization, (b) excitation.

as can be seen in Fig. 3. In contrast to [41], the extension of the $b$ range is well feasible with the present approach due to its seemingly higher efficiency. In all calculations 30 different $b$ values are considered whereas the maximal $b$ increases from 15 to 30 a.u. from the lowest to the highest impact energies. The spacing between the $b$ values increases with $b$ in order to sufficiently resolve the inner region. Figure 3(b) shows for example that a range of $b \leq 10$ a.u. is not large enough for calculating the excitation cross section for an impact energy of $E=125 \mathrm{keV}$. On the other hand in the case of ionization a range of $b \leq 5$ a.u. is according to Fig. 3(a) already sufficient.

The shape of $\sigma_{\text {exc }}$ in Fig. 2(b) is similar to that of $\sigma_{\text {ion }}$ in Fig. 2(a), although a little less symmetric. The absolute height of the maximum is, however, about a factor 4 larger for excitation than for ionization. This factor actually is minimal around the maximum and enlarges to about 6.5 toward the smallest and largest impact energies covered in the present work. The positions of the maxima are around 40 and $25 \mathrm{keV}$ for ionization and excitation, respectively. This is similar to the findings for hydrogen atoms but larger than for alkali-metal atom targets [31]. Calculations for $\bar{p}+\mathrm{H}_{2}$ [38,51] in which the target was described by an atomic effective one-electron model [40] yielded maxima for ionization and excitation which lie at lower and higher energies, respectively, than the present maxima for $\mathrm{H}_{2}^{+}$. 

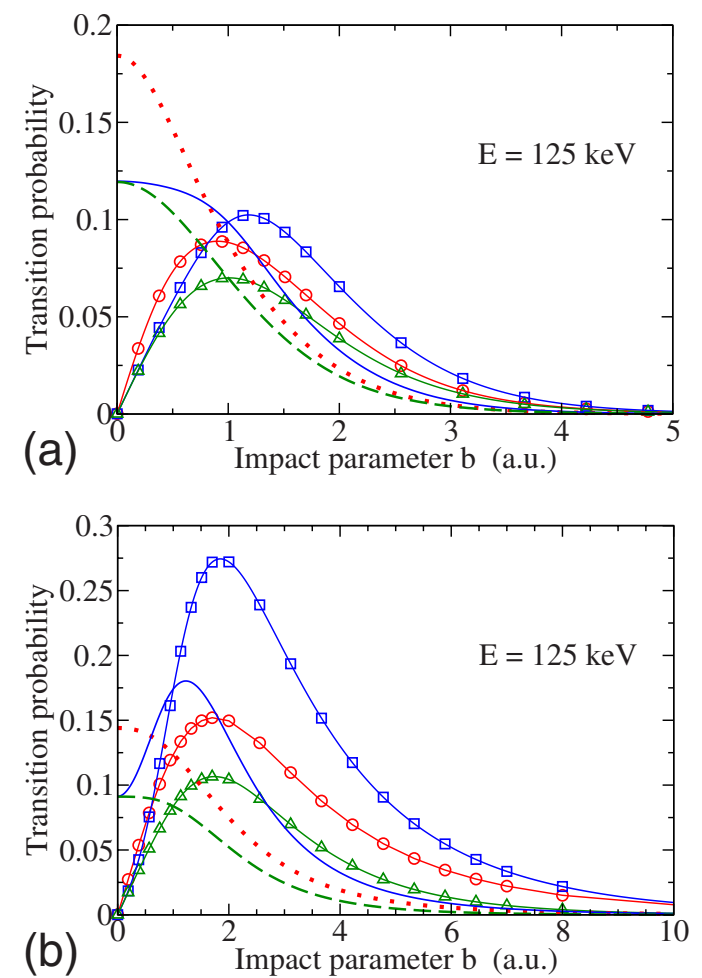

FIG. 3. (Color online) The transition probability $p(b)$ and $b p(b)$ - weighted with $b$ - for $\bar{p}+\mathrm{H}_{2}{ }^{+}$collisions as a function of the impact parameter $b$ for different molecular orientations and $E$ $=125 \mathrm{keV} \cdot p(b)$ : red dotted curve, $(\Theta, \Phi)=(0,0)$; blue solid curve, $(\pi / 2,0)$; green dashed curve, $(\pi / 2, \pi / 2) . b p(b)$ : red circles, $(0,0)$; blue squares, $(\pi / 2,0)$; green triangles, $(\pi / 2, \pi / 2)$. (a) Ionization, (b) excitation.

\section{Dependence on the molecular orientation}

The $\sigma_{\text {ion }}(\Theta, \Phi)$ and $\sigma_{\text {exc }}(\Theta, \Phi)$ as given in Eqs. (31) and (32) are presented in Figs. 4(a) and 4(b), respectively, as a function of the impact energy together with the orientationally averaged FC cross sections for comparison. The three perpendicular orientations (i), (ii), and (iii) are considered.

It can be said that the curves differ considerably for different orientations as well as from the orientationally averaged curve for both ionization and excitation. Similarities in the dependence on $E$, however, can be found for the same orientation between curves for ionization and excitation. Thereby, curve (ii) and (iii) share qualitatively a similar behavior while quantitatively the latter is for most energies clearly larger than the former.

For very low energies the curves for (ii) and (iii) seem to coincide for ionization $(E<2 \mathrm{keV})$ as well as for excitation $(E<1 \mathrm{keV})$. For these low energies the transition probability in Eq. (11) is only nonvanishing for very small $b$. The differences between the trajectories of the $\bar{p}$ for (ii) and (iii) increase with $b$ but become negligible for small $b$. This can be seen from Eqs. (16)-(18) which go for both orientations (ii) and (iii) in the limit $b \rightarrow 0$ over to $R_{x^{\prime}}=-v t, R_{y^{\prime}}=0$, and $R_{z^{\prime}}=0$. Consequently, the curves of the transition probabilities in Figs. 3(a) and 3(b) for (ii) and (iii) merge for $b \rightarrow 0$. These two curves are, on the other hand, most different around $b \approx 1$ a.u. where the trajectories of orientation (iii) encounter the position of the $\mathrm{H}_{2}^{+}$nuclei.
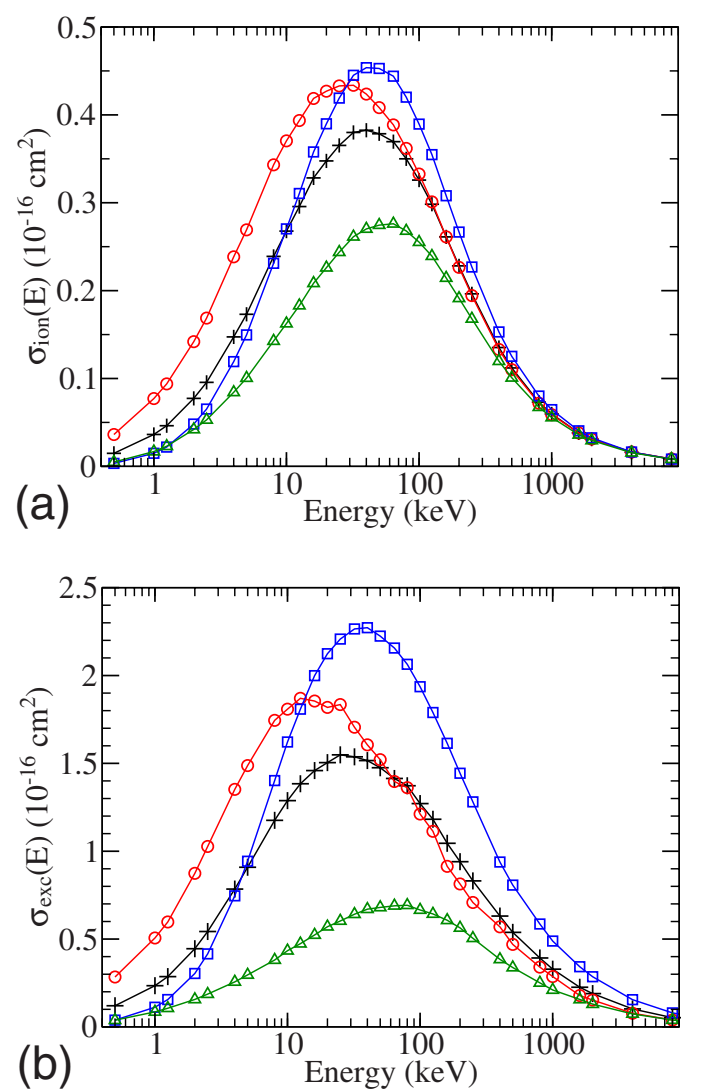

FIG. 4. (Color online) Ionization cross section $\sigma_{\text {ion }}$ and excitation cross section $\sigma_{\text {exc }}$ for $\bar{p}+\mathrm{H}_{2}^{+}$as a function of the antiproton impact energy for different molecular orientations. Black pluses, orientationally averaged; red circles, $(\Theta, \Phi)=(0,0)$; blue squares, $(\pi / 2,0)$; green triangles, $(\pi / 2, \pi / 2)$. (a) Ionization, (b) excitation.

For high energies $E>100 \mathrm{keV}$, on the other hand, the equality

$$
2 \sigma_{\text {ion }}(0,0)=\sigma_{\text {ion }}(\pi / 2,0)+\sigma_{\text {ion }}(\pi / 2, \pi / 2)=2 \sigma_{\text {ion }}
$$

holds with only about $1 \%$ deviation. In the case of excitation another equality,

$$
\sigma_{\text {exc }}(\pi / 2,0)=\sigma_{\text {exc }}(0,0)+\sigma_{\text {exc }}(\pi / 2, \pi / 2)=1.5 \sigma_{\text {exc }},
$$

holds already for $E \geq 50 \mathrm{keV}$ with the same accuracy as the one for ionization except for the energies $160 \mathrm{keV} \leq E$ $\leq 250 \mathrm{keV}$ where the deviation is of the order of $5 \%$. $\sigma_{\text {exc }}(0,0)$ shows some structures for energies above the maximum in contrast to the orientations (ii) and (iii).

For even higher energies $E \geq 2000 \mathrm{keV}$ all ionization curves seem to coincide. In the case of excitation $\sigma_{\text {exc }}(0,0)$ $\approx \sigma_{\text {exc }}(\pi / 2, \pi / 2)$ holds for $E \geq 4000 \mathrm{keV}$. The behavior at high energies can be explained by the fact that the contribution to excitation and ionization from distant encounters, i.e., larger $b$ values, increases with $E$. At larger distances from the center the electron is exposed to a quasicentral potential and the near-field details including the spatial distribution of the nuclei are not that much resolved any more. This is especially true for the orientations (i) and (iii), as can be nicely 
seen in Fig. 4(b), for which the molecular axis lies in the plane perpendicular to $\mathbf{b}$. This is in contrast to (ii) where the molecular axis is parallel to $\mathbf{b}$ and therefore the minimal distance between the antiproton and one of the $\mathrm{H}_{2}{ }^{+}$nuclei is smaller.

The observed dependence on the orientation at high energies suggests that ionization and excitation for $\bar{p}+\mathrm{H}_{2}{ }^{+}$can be described in accordance with Eqs. (33) and (34) surprisingly well with only one orientation, i.e., for ionization and $E$ $\geq 100 \mathrm{keV}$ by $\sigma_{\text {ion }}=\sigma_{\text {ion }}(0,0)$ and for excitation and $E$ $\geq 50 \mathrm{keV}$ by $\sigma_{\text {exc }}=2 / 3 \sigma_{\text {exc }}(\pi / 2,0)$. It also means that for $E \geq 100 \mathrm{keV}$ an appropriate one-center model potential might be sufficient in particular for the description of the ionization process. For these high energies satisfactory results were obtained in calculations for $\bar{p}+\mathrm{H}_{2}$ collisions $[38,51]$ using a one-center one-electron model for the description of the $\mathrm{H}_{2}$ molecule [40]. The calculations reproduced the experimental data [1,33] for $E \geq 90 \mathrm{keV}$. For lower energies the mentioned $\bar{p}+\mathrm{H}_{2}$ results resemble qualitatively those for the orientation (i) which separate from the orientationally averaged curves in Fig. 4 for $E<100$. Note, only one kind of trajectory is possible with the employed $\mathrm{H}_{2}$ model potential due to its atomic (spherical-symmetric) character. This kind of trajectory is practically the same as the one for the orientation (i) in the molecule-fixed frame.

Finally, it is interesting to note that the knowledge of the results for maximally three perpendicular orientations appears to be sufficient to accurately reproduce the total angular-integrated ionization cross section although the three curves differ considerably.

\section{Production of $\mathrm{H}^{+}$in $\bar{p}+\mathrm{H}_{2}^{+}$and $\bar{p}+\mathrm{H}_{2}$}

$$
\text { 1. } \bar{p}+\mathrm{H}_{2}^{+}
$$

In collisions of $\bar{p}$ with $\mathrm{H}_{2}{ }^{+}$three main mechanisms lead to the production of $\mathrm{H}^{+}$. First, ionization of the target

$$
\bar{p}+\mathrm{H}_{2}^{+} \rightarrow \bar{p}+\mathrm{H}^{+}+\mathrm{H}^{+}+e^{-},
$$

second, electronic excitation followed eventually by dissociation

$$
\bar{p}+\mathrm{H}_{2}^{+} \rightarrow \bar{p}+\mathrm{H}+\mathrm{H}^{+},
$$

third, direct dissociation which is not included in the present approach. At very low energies the replacement of the electron by the $\bar{p}$ may also become important. However, these energies lie beyond the scope of the present work [52,53]. As can be seen from Eqs. (35) and (36) two $\mathrm{H}^{+}$are produced in the ionization process while it is only one for excitation. The cross section for $\mathrm{H}^{+}$production due to ionization and excitation is therefore given by the sum $2 \sigma_{\text {ion }}+\sigma_{\text {exc }}$. It follows from the present results (cf. Table IV and Fig. 2) that the absolute contribution to this sum in $\bar{p}+\mathrm{H}_{2}{ }^{+}$collisions originates, however, only from a third to a quarter from ionization depending on whether the impact energy is close to or away from the maximum, respectively.

\section{2. $\bar{p}+\mathrm{H}_{2}$}

While no experimental data are available for $\bar{p}+\mathrm{H}_{2}{ }^{+}$collisions measurements of the ionization and the $\mathrm{H}^{+}$production cross sections have been performed for $\bar{p}+\mathrm{H}_{2}$ [1]. The present results obtained for $\bar{p}+\mathrm{H}_{2}{ }^{+}$collisions shall be used to learn more about the different production mechanisms of the measured $\mathrm{H}^{+}$cross section for $\bar{p}+\mathrm{H}_{2}$ which has not been explained theoretically so far. The analysis is done by applying the IEV as introduced in Sec. I. It was used by Wehrman et al. [22] for the description of double ionization in $\bar{p}+\mathrm{He}$ collisions. In the IEV double ionization is considered as a two-step process and the cross section is obtained by using the product of transition probabilities from (effective) oneelectron calculations only.

For the description of the first step the single-electron ionization probabilities $p_{\text {ion }}^{\mathrm{H}_{2}}$ for $\bar{p}+\mathrm{H}_{2}$ are calculated as explained in [38]. Therein, a simple one-center one-electron model potential for the description of the $\mathrm{H}_{2}$ target [40] was used which reproduces experimental ionization and excitation data well for $E \geq 90 \mathrm{keV}$.

In the second step $\bar{p}+\mathrm{H}_{2}{ }^{+}$collisions can contribute in two ways to the cross section for $\mathrm{H}^{+}$production (in contrast to $\bar{p}+\mathrm{He}^{+}$in double ionization of helium). They are given in Eqs. (35) and (36) each having the probability $p_{\text {ion }}^{\mathrm{H}^{+}}$and $p_{\text {exc }}^{\mathrm{H}^{+}}$, respectively. Therefore, both cross sections, $\sigma_{\mathrm{di}}$ for double ionization and $\sigma_{\mathrm{ie}}$ for ionization followed by excitation of an $\mathrm{H}_{2}$ target,

$$
\begin{gathered}
\sigma_{\mathrm{di}}=2 \pi \int p_{\mathrm{ion}}^{\mathrm{H}_{2}}(b) p_{\text {ion }}^{\mathrm{H}_{2}^{+}}(b) b \mathrm{~d} b, \\
\sigma_{\text {ie }}=2 \pi \int p_{\mathrm{ion}}^{\mathrm{H}_{2}}(b) p_{\text {exc }}^{\mathrm{H}_{2}^{+}}(b) b \mathrm{~d} b,
\end{gathered}
$$

are considered in accordance with the IEV.

Furthermore, all doubly-exited electronic states of $\mathrm{H}_{2}$ are in principle autoionizing. But it is also possible that the doubly-excited $\mathrm{H}_{2}$ dissociates before an electron is ejected. The description of this channel is clearly very subtle and has been studied in detail with a considerable effort for the excitation of $\mathrm{H}_{2}$ by photons $[54,55]$. The double-excitation cross section $\sigma_{\mathrm{de}}$ for $\bar{p}+\mathrm{H}_{2}$ collisions,

$$
\sigma_{\mathrm{de}}=2 \pi \int p_{\mathrm{exc}}^{\mathrm{H}_{2}}(b) p_{\mathrm{exc}}^{\mathrm{H}_{2}}(b) b \mathrm{~d} b,
$$

is approximated using the independent particle model [56]. The single-excitation probabilities $p_{\text {exc }}^{\mathrm{H}_{2}}$ for $\mathrm{H}_{2}$ originate form the same calculations as the $p_{\text {ion }}^{\mathrm{H}_{2}}$ used in Eqs. (37) and (38).

In Fig. 5 the results for $\sigma_{\mathrm{di}}$ and $\sigma_{\mathrm{ie}}$ are presented together with the sum of these cross sections

$$
\sigma_{\mathrm{H}^{+}}=2 \sigma_{\mathrm{di}}+\sigma_{\mathrm{ie}},
$$

in which $\sigma_{\mathrm{di}}$ is counted twice since two $\mathrm{H}^{+}$are produced in the double ionization of $\mathrm{H}_{2}$. Also given are the measured cross sections for the total $\mathrm{H}^{+}$production in $\bar{p}+\mathrm{H}_{2}$ by Hvelplund et al. [1] and for dissociative ionization for $p+\mathrm{H}_{2}$ by Shah and Gilbody [36], where the latter includes $\sigma_{\mathrm{di}}, \sigma_{\mathrm{ie}}$ and transfer ionization.

For all energies considered in Fig. $5 \sigma_{\text {di }}$ lies below $\sigma_{\text {ie }}$ by about a factor $\lesssim 2$. Therefore, both mechanisms in the $\mathrm{H}^{+}$ production as given in Eqs. (35) and (36) contribute with a 


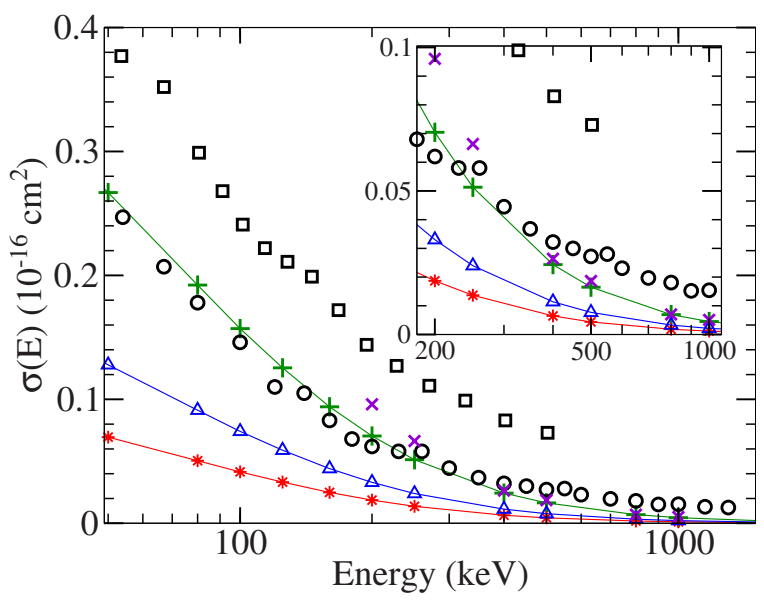

FIG. 5. (Color online) Cross sections leading to the production of $\mathrm{H}^{+}$in collisions with $\mathrm{H}_{2}$ targets as a function of the projectile energy. $\bar{p}$ impact. Present results. $\bar{p}$ : red stars, double ionization $\sigma_{\mathrm{di}}$; blue triangles, ionization and excitation $\sigma_{\text {ie }}$; green plus, summed $\mathrm{H}^{+}$ production $\sigma_{\mathrm{H}^{+}}=2 \sigma_{\mathrm{di}}+\sigma_{\mathrm{ie}} . p$ : violet $\mathrm{X}, \mathrm{H}^{+}$production $\sigma_{\mathrm{H}^{+}}$. Experimental data: black squares, total $\mathrm{H}^{+}$production for $\bar{p}+\mathrm{H}_{2}$ [1]; black circles, dissociative ionization for $p+\mathrm{H}_{2}$ [36]. The inset shows a high-energy cutout of the same data.

comparable amount of $\mathrm{H}^{+}$in $\sigma_{\mathrm{H}^{+}}$. Although the measured data for $\bar{p}+\mathrm{H}_{2}$ have a similar slope than the present $\sigma_{\mathrm{H}^{+}}$the latter is shifted down relative to the experimental results by between $\approx 10^{-17} \mathrm{~cm}^{2}$ for the lowest and $\approx 6 \times 10^{-18} \mathrm{~cm}^{2}$ for the highest energies in Fig. 5. The cross section for double excitation $\sigma_{\mathrm{de}}$ which is not included in $\sigma_{\mathrm{H}^{+}}$is of the order of approximately $10 \%$ of $\sigma_{\mathrm{H}^{+}}$in the whole energy range. This means that the three mechanisms for the production of $\mathrm{H}^{+}$ described with the employed models are not sufficient in order to reproduce the experimental data of Hvelplund et al. [1].

Note, the curves given in Fig. 5 are calculated with the FC data presented before which implies that the internuclear distances of the $\mathrm{H}_{2}{ }^{+}$is set to $R_{\text {nuc }}=2.05$ a.u. Under the assumption that the time between the first and the second step in the IEV is too short to allow for a change of the internuclear distance the $\bar{p}+\mathrm{H}_{2}{ }^{+}$collisions are also calculated for $R_{\text {nuc }}$ $=1.4478$ a.u. which is the expectation value of $\mathrm{H}_{2}$. This leads to an increase of the binding energy and therefore to a decrease of the ionization and excitation cross sections of $\bar{p}+\mathrm{H}_{2}{ }^{+}$. Consequently, the results for $R_{\text {nuc }}=1.4478$ a.u., which are not shown in Fig. 5, become even smaller and reproduce those for $R_{\text {nuc }}=2.05$ a.u. from about $80 \%$ for $E$ $=50 \mathrm{keV}$ to $90 \%$ for $E=1000 \mathrm{keV}$.

The experimental data for dissociative ionization in $p$ $+\mathrm{H}_{2}$ collisions by Shah and Gilbody also have a similar slope as the present results. Note, in contrast to the measured $\bar{p}$ data, their absolute values are comparable with those of the present $\sigma_{\mathrm{H}^{+}}$for $\bar{p}$.

Additionally, $\sigma_{\mathrm{H}^{+}}$results for $p$ collisions with $\mathrm{H}_{2}$ are calculated and shown in Fig. 5. They are obtained exactly in the same way as described for $\bar{p}$ impact only that the projectile charge $Z_{p}$ in the interaction potential [cf. Eq. (8)] is set to +1 instead of -1 for $\bar{p}$. Although the present approach does not distinguish between ionization and electron capture by the proton, the $p$ results are still meaningful for high energies since the cross section for electron capture for $p+\mathrm{H}_{2}$ is negligible for $E \geq 200 \mathrm{keV}$ [37,57]. In general, the present data for $\bar{p}$ and $p$ impact are very similar and practically the same for $E \geq 400 \mathrm{keV}$ both being close to the experimental proton results. This means that an obvious difference of the $\mathrm{H}^{+}$production between $\bar{p}$ and $p$ impacts for high energies as measured experimentally and suggested by the double-ionization cross sections for He targets cannot be reproduced by the present study.

Within the employed two-step model it might be crucial to consider an orientational dependence also for the $p_{\text {ion }}^{\mathrm{H}_{2}}$ as is done in the present method. That way, the probability $p_{\text {ion }}^{\mathrm{H}_{2}}$ can be multiplied first for each orientation individually with the probabilities for $\mathrm{H}_{2}^{+}$, as in the Eqs. (37) and (38) and being only afterward orientationally averaged. In the case that the dependence on $E$ of the $p_{\text {ion }}^{\mathrm{H}_{2}}$ for the three orientations is similar to that of the $\mathrm{H}_{2}^{+}$target as shown in Fig. 4 this might lead to a sizable effect on $\sigma_{\mathrm{di}}$ and $\sigma_{\mathrm{ie}}$ and therefore also on $\sigma_{\mathrm{H}^{+}}$

However, an advanced treatment of the $\bar{p}+\mathrm{H}_{2}$ collision would be eligible which includes at least a two-electron description of the target in contrast to what has been done in [38] in order to resolve the discrepancy which appears between experiment and theory.

\section{SUMMARY AND CONCLUSION}

Time-dependent close-coupling calculations using a spectral expansion are performed in order to determine ionization and excitation cross section for $\bar{p}+\mathrm{H}_{2}{ }^{+}$collisions in a broad energy range from $0.5 \mathrm{keV}$ to $10 \mathrm{MeV}$. For the description of the target the Born-Oppenheimer approximation and a onecenter expansion of the molecular potential are used. The collision process is treated within the impact parameter method as well as the Franck-Condon approximation. The transition probabilities are obtained by averaging the results for only three perpendicular orientations of the molecular axis with respect to the $\bar{p}$ trajectory instead of performing an integration over all orientations by what the numerical effort is reduced drastically. The use of symmetries for these three orientations leads to selection rules which further reduce the effort by about a factor 4 .

Extensive convergence studies assure that the final results do not depend on the expansion parameters. The present ionization cross section reproduces nicely the results calculated by Sakimoto [41] showing that the use of three perpendicular molecular orientations is sufficient. An extension of the range of the impact parameter $b$ in comparison with [41] is possible due to the less demanding calculations. The larger $b$ range allows for the determination of the excitation cross section. In general, the contribution to the total cross sections for a fixed impact energy differs considerably for the three perpendicular orientations. However, for high energies the cross sections for ionization $(E \geq 100 \mathrm{keV})$ and excitation $(E \geq 50 \mathrm{keV})$ can already be generated with only one molecular orientation.

The cross sections for double ionization and ionization followed by excitation are studied as well as their contribu- 
tion to the $\mathrm{H}^{+}$production in $\bar{p}+\mathrm{H}_{2}$ collisions. A sequential two-step model also referred to as independent event model is employed to extract these cross sections only from (effective) single-electron transition probabilities. The transition probabilities for $\mathrm{H}_{2}{ }^{+}$targets are taken from the present study while those for single ionization of $\mathrm{H}_{2}$ are calculated according to [38]. The present results do not match and are smaller than the experimental data [1] from $50 \mathrm{keV}$ to $2 \mathrm{MeV}$. The present data are, however, very similar in the case that $p$
$+\mathrm{H}_{2}$ collisions [36] are considered. This motivates further experimental and theoretical work on $\bar{p}+\mathrm{H}_{2}$ collisions.

\section{ACKNOWLEDGMENTS}

The authors are grateful to BMBF (FLAIR Horizon), Stifterverband für die deutsche Wissenschaft, and to the Fonds der Chemischen Industrie for financial support.
[1] P. Hvelplund, H. Knudsen, U. Mikkelsen, E. Morenzoni, S. P. Møller, E. Uggerhøj, and T. Worm, J. Phys. B 27, 925 (1994).

[2] L. H. Andersen, P. Hvelplund, H. Knudsen, S. P. Møller, K. Elsener, K. G. Rensfelt, and E. Uggerhøj, Phys. Rev. Lett. 57, 2147 (1986).

[3] L. H. Andersen, P. Hvelplund, H. Knudsen, S. P. Møller, A. H. Sorensen, K. Elsener, K.-G. Rensfelt, and E. Uggerhoj, Phys. Rev. A 36, 3612 (1987).

[4] L. H. Andersen, P. Hvelplund, H. Knudsen, S. P. Møller, J. O. P. Pedersen, S. Tang-Petersen, E. Uggerhøj, K. Elsener, and E. Morenzoni, Phys. Rev. A 41, 6536 (1990).

[5] H. Knudsen and J. F. Reading, Phys. Rep. 212, 107 (1992).

[6] H. Knudsen, U. Mikkelsen, K. Paludan, K. Kirsebom, S. P. Møller, E. Uggerhøj, J. Slevin, M. Charlton, and E. Morenzoni, Phys. Rev. Lett. 74, 4627 (1995).

[7] H. Knudsen, H.-P. Kristiansen, H. Thomsen, U. Uggerhøj, T. Ichioka, S. Møller, N. Kuroda, Y. Nagata, H. Torii, H. Imao et al., Phys. Rev. Lett. 101, 043201 (2008).

[8] J. Azuma, N. Toshima, K. Hino, and A. Igarashi, Phys. Rev. A 64, 062704 (2001).

[9] J. C. Wells, D. R. Schultz, P. Gavras, and M. S. Pindzola, Phys. Rev. A 54, 593 (1996).

[10] G. Schiwietz, U. Wille, R. D. Muiño, P. D. Fainstein, and P. L. Grande, J. Phys. B 29, 307 (1996).

[11] K. A. Hall, J. F. Reading, and A. L. Ford, J. Phys. B 29, 6123 (1996).

[12] A. Igarashi, S. Nakazaki, and A. Ohsaki, Phys. Rev. A 61, 062712 (2000).

[13] K. Sakimoto, J. Phys. B 33, 3149 (2000).

[14] B. Pons, Phys. Rev. Lett. 84, 4569 (2000).

[15] B. Pons, Phys. Rev. A 63, 012704 (2000).

[16] X.-M. Tong, T. Watanabe, D. Kato, and S. Ohtani, Phys. Rev. A 64, 022711 (2001).

[17] N. Toshima, Phys. Rev. A 64, 024701 (2001).

[18] S. Sahoo, S. C. Mukherjee, and H. R. J. Walters, J. Phys. B 37, 3227 (2004).

[19] K. Sakimoto, J. Phys. B 37, 2255 (2004).

[20] A. Igarashi, S. Nakazaki, and A. Ohsaki, Nucl. Instrum. Methods Phys. Res. B 214, 135 (2004).

[21] M. Foster, J. Colgan, and M. S. Pindzola, Phys. Rev. Lett. 100, 033201 (2008).

[22] L. A. Wehrman, A. L. Ford, and J. F. Reading, J. Phys. B 29, 5831 (1996).

[23] J. F. Reading, T. Bronk, A. L. Ford, L. A. Wehrman, and K. A. Hall, J. Phys. B 30, L189 (1997).

[24] G. Bent, P. S. Krstić, and D. R. Schultz, J. Chem. Phys. 108,
1459 (1998).

[25] T. G. Lee, H. C. Tseng, and C. D. Lin, Phys. Rev. A 61, 062713 (2000).

[26] T. Kirchner, M. Horbatsch, E. Wagner, and H. J. Lüdde, J. Phys. B 35, 925 (2002).

[27] X.-M. Tong, T. Watanabe, D. Kato, and S. Ohtani, Phys. Rev. A 66, 032709 (2002).

[28] M. Keim, A. Achenbach, H. J. Lüdde, and T. Kirchner, Phys. Rev. A 67, 062711 (2003).

[29] D. R. Schultz and P. S. Krstić, Phys. Rev. A 67, 022712 (2003)

[30] S. M. S. Sahoo and H. Walters, Nucl. Instrum. Methods Phys. Res. B 233, 318 (2005).

[31] A. Lühr and A. Saenz, Phys. Rev. A 77, 052713 (2008).

[32] T. Kirchner, M. Horbatsch, and H. J. Lüdde, Phys. Rev. A 66, 052719 (2002).

[33] L. H. Andersen, P. Hvelplund, H. Knudsen, S. P. Møller, J. O. P. Pedersen, S. Tang-Petersen, E. Uggerh $\varnothing$ j, K. Elsener, and E. Morenzoni, J. Phys. B 23, L395 (1990).

[34] A. Adamo, M. Agnello, F. Balestra, G. Belli, G. Bendiscioli, A. Bertin, P. Boccaccio, G. C. Bonazzola, T. Bressani, M. Bruschi et al., Phys. Rev. A 47, 4517 (1993).

[35] M. Agnello, G. Belli, G. Bendiscioli, A. Bertin, E. Botta, T. Bressani, M. Bruschi, M. P. Bussa, L. Busso, D. Calvo et al., Phys. Rev. Lett. 74, 371 (1995).

[36] M. B. Shah and H. B. Gilbody, J. Phys B. 15, 3441 (1982).

[37] M. B. Shah, P. McCallion, and H. B. Gilbody, J. Phys B. 22, 3037 (1989).

[38] A. Lühr and A. Saenz, Phys. Rev. A 78, 032708 (2008).

[39] A. Lühr and A. Saenz, Phys. Rev. A 79, 042901 (2009).

[40] A. Lühr, Y. V. Vanne, and A. Saenz, Phys. Rev. A 78, 042510 (2008).

[41] K. Sakimoto, Phys. Rev. A 71, 062704 (2005).

[42] R. K. Janev, E. A. Solov'ev, and D. Jakimovski, J. Phys. B 28, L615 (1995).

[43] A. Apalategui, A. Saenz, and P. Lambropoulos, J. Phys. B 33, 2791 (2000).

[44] A. Apalategui, A. Saenz, and P. Lambropoulos, Phys. Rev. Lett. 86, 5454 (2001).

[45] A. Apalategui and A. Saenz, J. Phys. B 35, 1909 (2002).

[46] T. N. Chang and X. Tang, Phys. Rev. A 44, 232 (1991).

[47] T. N. Chang, in Many-body Theory of Atomic Structure and Photoionization, edited by T. N. Chang (World Scientific, Singapore, 1993), p. 213.

[48] B. H. Bransden and M. R. C. McDowell, Charge Exchange and the Theory of Ion-Atom Collisions (Clarendon, Oxford, 
1992).

[49] A. Saenz and P. Froelich, Phys. Rev. C 56, 2162 (1997).

[50] M. McGovern, D. Assafrão, J. R. Mohallem, C. T. Whelan, and H. R. J. Walters, Phys. Rev. A 79, 042707 (2009).

[51] A. Lühr and A. Saenz, Hyperfine Interact. (to be published).

[52] J. S. Cohen, Rep. Prog. Phys. 67, 1769 (2004).

[53] K. Sakimoto, Phys. Rev. A 69, 042710 (2004).

[54] F. Martín, J. Phys. B 32, R197 (1999).
[55] J. Fernandez and F. Martin, New J. Phys. 11, 043020 (2009). [56] H. J. Lüdde and R. M. Dreizler, J. Phys. B 16, 3973 (1983).

[57] M. E. Rudd, R. D. DuBois, L. H. Toburen, C. A. Ratcliffe, and T. V. Goffe, Phys. Rev. A 28, 3244 (1983).

[58] In (ii) $V^{(\phi)}$ changes at $R_{x^{\prime}}=0$ from 1 discontinuously to $(-1)^{M^{\prime}-M}$ what has to be taken into account during the calculation. 